\title{
Ambulatory respiratory rate trends identify patients at higher risk of worsening heart failure in implantable cardioverter defibrillator and biventricular device recipients: a novel ambulatory parameter to optimize heart failure management
}

\author{
Stephan Goetze • Yi Zhang • Qi An • Viktoria Averina • \\ Pier Lambiase • Richard Schilling • \\ Hans-Joachim Trappe • Siegmund Winter • \\ Nicholas Wold • L jubomir Manola • Dries Kestens
}

Received: 13 November 2014 / Accepted: 4 February 2015 / Published online: 12 April 2015

(C) Springer Science+Business Media New York 2015

\begin{abstract}
Purpose Respiratory distress is the primary driver for heart failure (HF) hospitalization. Implantable pacemakers and defibrillators are capable of monitoring respiratory rate (RR) in ambulatory HF patients. We investigated changes in RR prior to HF hospitalizations and its near-term risk stratification power.

Methods NOTICE-HF was an international multi-center study. Patients were implanted with an implantable cardioverter defibrillator or cardiac resynchronization therapy defibrillator, capable of trending daily maximum, median, and minimum RR (maxRR, medRR, minRR). RR from 120 pa-
\end{abstract}

Clinical Trial Registration: URL: http://www.clinicaltrials.gov. Unique identifier: NCT01227785.

\author{
S. Goetze $(\bowtie)$ \\ German Heart Institute, Berlin, Germany \\ e-mail: Stephan-goetze@web.de \\ Y. Zhang $\cdot$ Q. An $\cdot$ V. Averina $\cdot$ N. Wold \\ Boston Scientific, St Paul, USA \\ P. Lambiase \\ Heart Hospital, London, UK \\ R. Schilling \\ Barts Health, London, UK \\ H.-J. Trappe \\ Marien Hospital, Herne, Germany \\ S. Winter \\ Public Elizabethinen Hospital, Linz, Austria \\ L. Manola $\cdot$ D. Kestens \\ Boston Scientific, Brussels, Belgium
}

tients with 9 months of follow-up was analyzed. One-tailed Student's $t$ test was used to compare RR values prior to HF events to baseline defined as 4 weeks prior to the events. A Cox regression model was used to calculate the hazard ratios (HR) for the 30-day HF hospitalization risk based on RR values in the preceding month.

Results Daily maxRR, medRR, and minRR were significantly elevated prior to HF events compared to baseline ( $\Delta$ maxRR $1.8 \pm 3.0 ; p=0.02 ; \Delta \operatorname{medRR}, 2.1 \pm 2.8 ; p=0.007 ; \Delta \operatorname{minRR}$, $1.5 \pm 2.1, p=0.008)$. Risk of experiencing HF events within 30-days was increased if the standard deviation of medRR over the preceding month was above $1.0 \mathrm{br} / \mathrm{min}(\mathrm{HR}=12.3$, $95 \%$ confidence interval (CI) $2.57-59, p=0.002$ ). The risk remained high after adjusting for clinical variables that differed at enrollment.

Conclusion Ambulatory daily respiratory rate trends may be a valuable addition to standard management for HF patients.

Keywords Implantable device $\cdot$ Respiratory rate $\cdot$ Heart failure $\cdot$ Risk stratification

\section{Introduction}

Respiratory distress is common in heart failure (HF) patients $[1,2]$ and is the primary driver for HF hospitalization. The ADHERE $^{\circledR}$ registry showed that $89 \%$ of patients presented with some type of dyspnea and $36 \%$ with dyspnea at rest at HF admission [3]. Respiratory distress is also associated with worse clinical outcomes. Respiratory symptoms such as breathlessness and orthopnea are significantly related to increased mortality and hospitalization [4, 5]. Rapid respiratory 
rate (RR) has been shown to be a predictor of increased risk for hospitalization and mortality [6, 7]. A method for reliably detecting early respiratory distress may allow identification of patients at high risk of decompensation and thereby trigger timely patient care and intervention.

When patients complain of dyspnea, their breathing is often rapid and shallow [8-11]. Although HF patients have a higher respiratory rate than normal controls, both during stable periods [11] and during acute decompensation [12], relatively, little is known about respiratory rate changes leading up to worsening HF largely due to the difficulty of ambulatory respiratory monitoring. Charachet al. [8] demonstrated that respiratory rate often increased during the evolving stage of cardiopulmonary edema and decreased during its resolution. Moreover, respiratory rate varied with the severity of edema during both the evolving and the resolution phase.

Many HF patients are implanted with cardioverter defibrillators (ICD), or cardiac resynchronization therapy defibrillators (CRT-D), some of which are capable of monitoring respiration in an ambulatory setting. The purpose of this analysis is to evaluate respiratory rate trends before HF hospitalization and assess its prognostic value in identifying impending $\mathrm{HF}$ hospitalizations.

\section{Methods}

NOTICE-HF (Next Generation INCEPTA ${ }^{\mathrm{TM}}$ ICD and CRTD Field Following Study: Respiratory Rate Trend Evaluation in Heart Failure Patients) was a prospective international multi-center study. The purpose of the study was to evaluate and document appropriate clinical performance of the INCE PTA $^{\mathrm{TM}}$ ICD and CRT-D systems, and to demonstrate the clinical relevance of chronic ambulatory daily respiratory rate trend (RRT) in HF patients. Twenty-five centers from 13 countries in Europe and Asia participated in the study. The study protocol was approved by appropriate regulatory bodies and the ethical committee of each center as applicable. The study was registered on clinicaltrials.gov (identifier NCT01227785).

Patients who were 18 or older, willing to participate and indicated for ICD or CRT-D were implanted with INCE $\mathrm{PTA}^{\mathrm{TM}}$. After enough ICD patients were enrolled for clinical performance evaluation, only NYHA Class III patients were enrolled in the study to maximize the number of HF events captured during follow-up. Patients who received routinely scheduled intravenous (IV) inotropic therapy in the last 90 days, prescribed positive airway pressure therapy, had a life expectancy of less than 1 year, or were NYHA class IV during the last 4 weeks were excluded from the study.

The device is capable of collecting three daily RRT metrics up to a year: maximum (maxRR), median (medRR), and minimum (minRR). The device and clinical data were collected at
1-, 3-, 6- and 9-month follow-up visits. A HF event was defined as follows: patients had signs and/or symptoms of $\mathrm{HF}$ and one of the following: (1) received unscheduled IV therapy without hospital admission, (2) was admitted to the hospital with a calendar date change and received additional HF treatment. All HF events were reported through case report forms and adjudicated by a Boston Scientific internal clinician who was blinded to the device data.

\section{Statistical analysis}

Mean and standard deviations (SDs) of the three RRT metrics during a 9-month follow-up were calculated for each patient. In patients who experienced a HF event (HFE group), this calculation excluded data from 30 days prior to the event to 14 days after the event. In patients who completed the study without a HF event (nHFE group), all data were used. Student's $t$ test was used to compare the two groups.

We also retrospectively evaluated changes in RRT ( $\Delta$ maxRR, $\Delta$ medRR, $\Delta$ minRR) during the 4-week period before the HF event in HFE group. For this analysis, a 5-day moving average filter was applied to each trend parameter. One-tailed Student's $t$ test was used to evaluate if values at 3 weeks, 2 weeks, 1 weeks and 1-day before HF events were significantly elevated compared to the baseline defined as 4 weeks prior to a HF event. Events with less than 28 days of pre-event data were excluded from the analysis. A $p$ value $<0.025$ was regarded statistically significant.

We further evaluated the potential of using RRT to assess the 30-day risk of experiencing worsening HF. Data were divided into sequential 30 -day periods beginning at enrollment. Six risk indices were calculated for each 30-day period (evaluation window) and compared to a threshold to determine if the patient was at low or high risk for HF events in the next 30-day period (observation window). An empirical threshold was chosen to maximize the separation between two groups. The incidence of HF events in each observation window was calculated in low- and high-risk groups and compared using a negative binomial test (Fig. 1). A proportional means model was used to quantify the association between each monthly HF event risk and the percentage of event-free patients observed within the corresponding observation window. Repeated measurements within patient are accounted using the proportional means model with sandwich covariance estimation [13]. To calculate the event-free period, only the first HF event in each observation window was included. Hazard ratios (HRs), $95 \%$ confidence intervals (CIs) and $p$ values were reported using the proportional means model in both contexts before and after adjusting for clinical variables that differed at enrollment.

The performance of risk indices that were statistically significant between high and low risk groups $(p<0.05)$ were 
Fig. 1 A schematic plot of the HF risk analysis schedule. Each monthly HF event risk assessment is calculated using RR collected during a 30-day evaluation period and is compared to the number of protocol-defined HF events during a subsequent 30-day observation period
Whole follow-up period of a patient

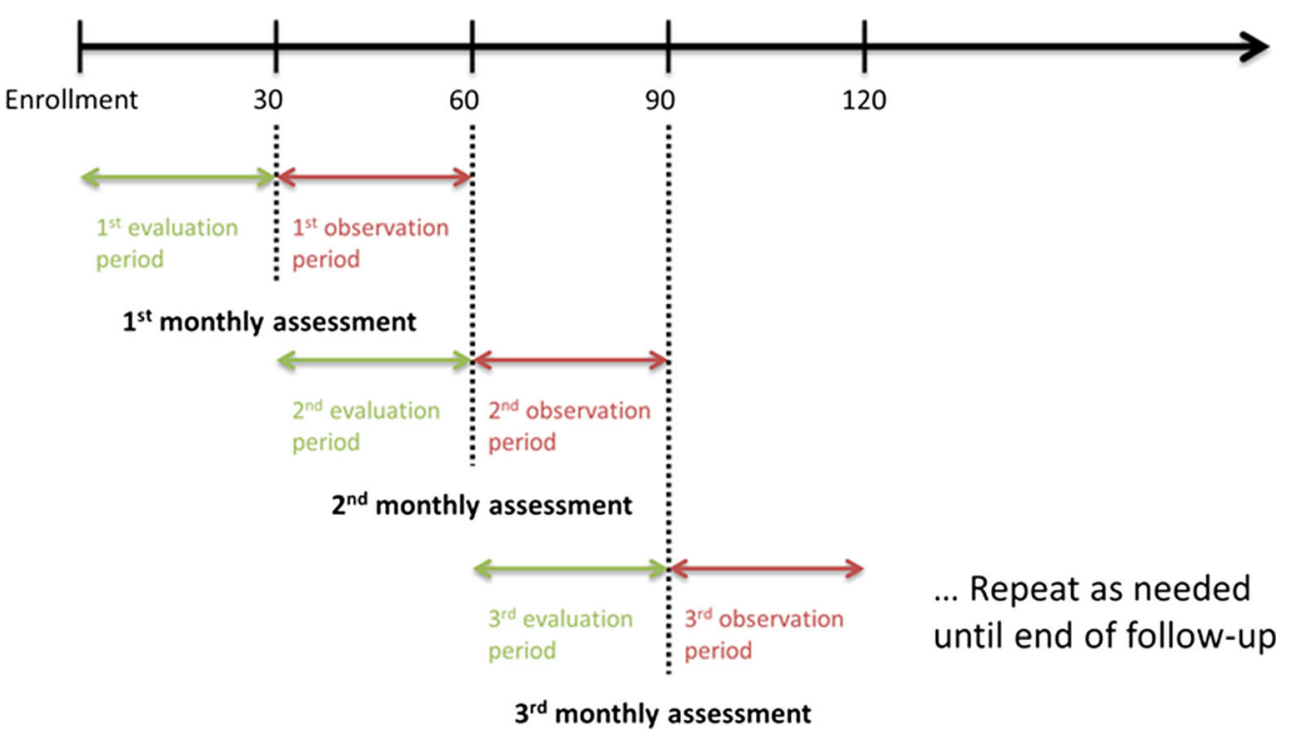

further evaluated using a binary classification test. A risk assessment was excluded from the analysis if either its evaluation window had less than 14 days of data or its observation window was shorter than 30 days. A high HF event risk assessment was classified as true positive (TP) if there was at least one HF event within its observation window, otherwise it was classified as false positive (FP). Low HF event risk assessments with HF event-free observation windows were classified as true negative (TN), otherwise they were classified as false negative (FN). Sensitivity was calculated as the number of TP divided by the number of observation windows with at least one HF event. Specificity was calculated as the number of TN divided by the number of HF event-free observation windows. Positive predictive value (PPV) was calculated as the number of TP divided by the number of high HF event risk assessments. Negative predictive value (NPV) was calculated as the number of TN divided by the number of low HF event risk assessments.

\section{Results}

There were 120 patients enrolled and implanted with the device in the study between November 2010 and February 2011. Seventeen HF events occurred in 13 patients during the $9.2 \pm$ 0.5 months of follow-up, 4 of which occurred within 28 days of implant. Patient demographics, medical history, and medication for the HFE and nHFE groups are shown in Table 1, where continuous variables are presented as means and SDs, and categorical variables are presented as frequencies and percentages. All patients enrolled had at least New York Heart Association (NYHA) class I HF. While all patients who had experienced a HFE were class III at enrollment, NYHA class and LVEF were not significantly different between the HFE and nHFE group. Patients in the HFE group had a higher resting heart rate and a lower blood pressure, as well as more cardiac surgery, anemia, and different prior hospitalization status.

Mean and SD of RRTs during the study are summarized in Fig. 2 for each group. Mean of all three daily RRT metrics was similar in HFE and nHFE groups. SD of daily medRR was significantly higher in the HFE group $(1.5 \pm 0.4$ vs. $1.1 \pm 0.4$ breaths $(\mathrm{br}) / \mathrm{min}, p<0.01)$, while SD of daily maxRR and minRR was not.

Figures 3 and 4 show case examples of the three RRT metrics in patients with and without HF events. Figure 3 shows RRTs in a patient without HF admissions. All three RRT metrics were stable during the study. Consistent with the group analysis of SDs, the case data illustrate the greater day-to-day variation in maxRR and minRR (shown in the background of figures using dotted lines) as compared to medRR. Figure 4 shows RRTs in a patient with a HF admission. All three RRT metrics were clearly elevated during the month before the admission.

Aggregated changes in three RRT metrics during a 4-week period prior to HF events are shown in Fig. 5. A ramp-up pattern was observed leading up to HF events. All three metrics were significantly elevated the week before HF events, compared to a baseline of 4 -week before HF events ( $\Delta$ maxRR, $1.8 \pm 3.0 ; p=0.02 ; \Delta$ medRR, $2.1 \pm 2.8 ; p=0.007$; $\Delta \operatorname{minRR}, 1.5 \pm 2.1, p=0.008$ ).

In order to assess the risk of experiencing HF events in next 30 days, monthly RR assessments were evaluated. There were 923 total HF event risk assessments (7.7 \pm 2.4 assessments per patient; range 1 to 10 per patient). Of all the monthly assessments, 18 assessments in 11 patients had less than 14 days of 
Table 1 Patient baseline characteristics

\begin{tabular}{|c|c|c|c|}
\hline Patient baseline characteristic & $\operatorname{nHFE}(N=107)$ & $\operatorname{HFE}(\mathrm{N}=13)$ & $p$ value \\
\hline Age at implant (years) & $65.1 \pm 10.6$ & $70.8 \pm 7.7$ & 0.07 \\
\hline Gender $(\mathrm{M} / \mathrm{F})[N(\%)]$ & $89(83) / 18(17)$ & $11(85) / 2(15)$ & 0.90 \\
\hline Device (CRT-D/ICD) [N (\%)] & $67(63) / 40(37)$ & $10(77) / 3(23)$ & 0.31 \\
\hline NYHA class (I/II/III) [N (\%)] & $3(3) / 10(9) / 94(88)$ & $0(0) / 0(0) / 13(100)$ & 0.41 \\
\hline $\operatorname{LVEF}(\%)$ & $27.7 \pm 9.9$ & $25.8 \pm 6.5$ & 0.51 \\
\hline QRS duration (ms) & $136 \pm 35$ & $134 \pm 24$ & 0.81 \\
\hline Heart rate at rest (bpm) & $70 \pm 16$ & $82 \pm 20$ & 0.01 \\
\hline Body mass index $\left(\mathrm{kg} / \mathrm{m}^{2}\right)$ & $28.1 \pm 5.3$ & $27.8 \pm 4.0$ & 0.80 \\
\hline Systolic blood pressure (mmHg) & $125 \pm 18$ & $116 \pm 23$ & 0.07 \\
\hline Diastolic blood pressure (mmHg) & $73 \pm 10$ & $67 \pm 8$ & 0.04 \\
\hline \multicolumn{4}{|l|}{ Medical history $[N(\%)]$} \\
\hline Ischemic/dilated cardiomyopathy & $53(50) / 47(44)$ & $9(69) / 4(31)$ & 0.90 \\
\hline Coronary artery disease & $48(45)$ & $8(62)$ & 0.26 \\
\hline Hypertension & $66(62)$ & $9(69)$ & 0.60 \\
\hline History of cardiac surgery & $33(31)$ & $8(62)$ & 0.04 \\
\hline Diabetes & $36(34)$ & $5(38)$ & 0.73 \\
\hline Renal disease & $25(23)$ & $5(38)$ & 0.24 \\
\hline COPD & $15(14)$ & $2(15)$ & 0.89 \\
\hline Asthma & $6(6)$ & $1(8)$ & 0.76 \\
\hline Anemia & $0(0)$ & $1(8)$ & 0.004 \\
\hline Pulmonary hypertension & $8(7)$ & $5(38)$ & $<0.001$ \\
\hline Hyperlipidemia & $42(39)$ & $6(46)$ & 0.63 \\
\hline Hospitalizations in last 12 months (yes/no/unknown) & $31(29) / 64(60) / 12(11)$ & $3(23) / 4(31) / 6(46)$ & 0.004 \\
\hline \multicolumn{4}{|l|}{ Medication $[N(\%)]$} \\
\hline Beta blocker & $94(88)$ & $12(92)$ & 0.64 \\
\hline Diuretics & $90(84)$ & $12(92)$ & 0.43 \\
\hline ACE inhibitor & $77(72)$ & $10(77)$ & 0.71 \\
\hline Aldosterone antagonist & $45(42)$ & $6(46)$ & 0.78 \\
\hline Angiotensin receptor blocker & $23(21)$ & $3(23)$ & 0.90 \\
\hline Anti-arrhythmic drugs & $11(10)$ & $2(15)$ & 0.58 \\
\hline Other & $50(47)$ & $5(38)$ & 0.57 \\
\hline
\end{tabular}

data for the calculation of the risk index and 105 additional assessments in 105 patients had less than 30 days in the following observation window. The remaining $800 \mathrm{HF}$ event risk assessments in 111 patients $(7.2 \pm 1.5$ per patient, range 2 to 10 , with $11 \mathrm{HF}$ events in 9 patients) were considered complete and were analyzed. A proportional means model with
Fig. 2 Mean and SD RR during the study (for the HFE group, data between 30 days prior to and 14 days post HF events were excluded from the analysis)
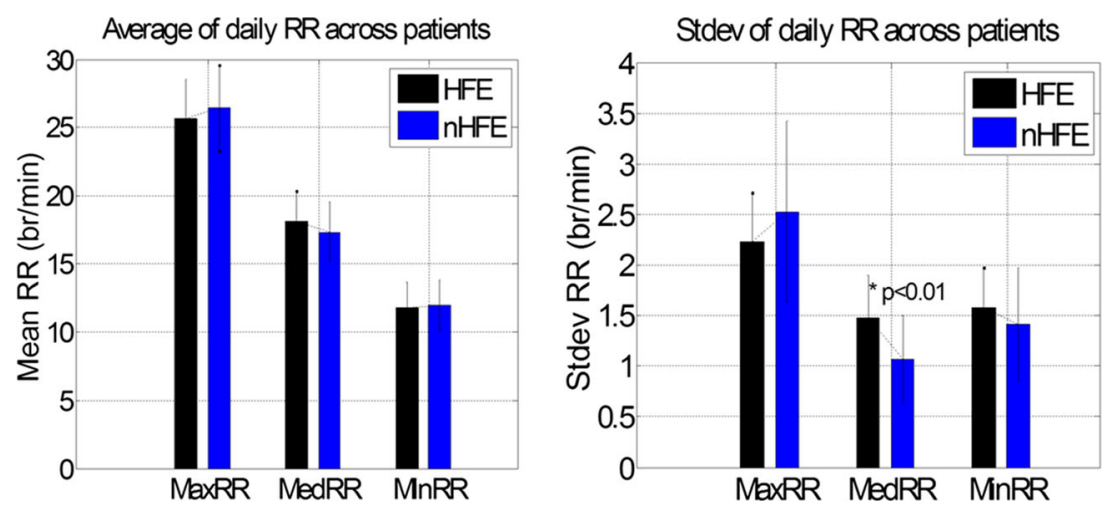
Fig. 3 Respiratory rates in a patient without $\mathrm{HF}$ admissions. From top to bottom: daily maximum, median, and minimum respiratory rates. Thin lines with markers: daily trends; thick lines: 5-day moving averages

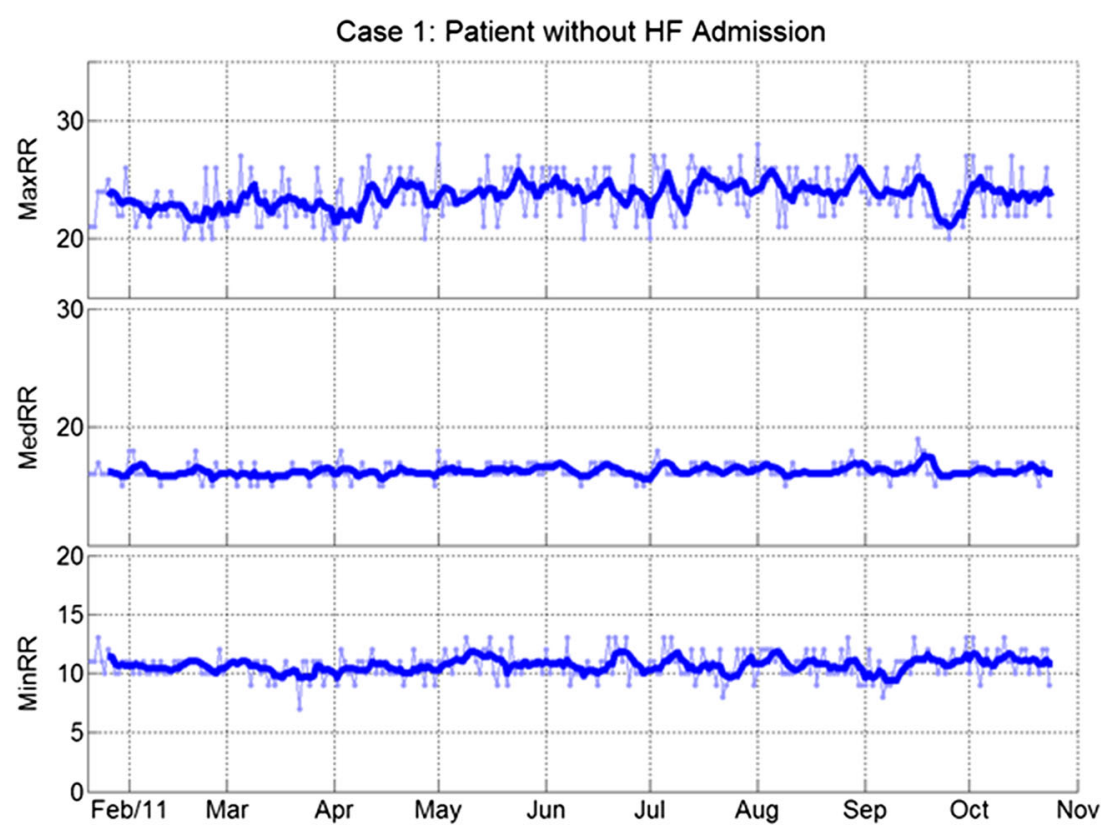

sandwich covariance estimation was used to assess the hazard ratio and showed significant separation between patients with high HF risk and those with low HF risk when using the SD of medRR. A threshold of $1.0 \mathrm{br} / \mathrm{min}$ was found to maximize the separation between two groups. Figure 6 shows the monthly assessment results from the proportional means model of event-free time using the SD of medRR. The proportional means model estimated a 12.3-fold increased risk of experiencing a HF event within the next month for patients with high risk indices ( $\mathrm{HR}=12.3,95 \% \mathrm{CI} 2.57-59, p=0.002)$. The risk remained high $(\mathrm{HR}=8.9,95 \% \mathrm{CI} 1.75-45, p=0.008)$ after adjusting for clinical variables that differed at enrollment, including heart rate at rest, diastolic blood pressure, history of cardiac surgery, anemia, and pulmonary hypertension. Event rates were also significantly higher in the high risk group and were 0.503 and 0.042 per patient-year in each group, respectively $(p=0.002)$.

Out of the $800 \mathrm{HF}$ risk assessments, 218 were in the high risk group (above the threshold) with nine HF events occurred during the observation windows and 582 in the low risk group (below the threshold) with two HF events in the observation windows. The risk stratification algorithm has a sensitivity of $81.8 \%$, a specificity of $73.5 \%$, a NPV of $99.7 \%$, and a PPV of $4.1 \%$. Out of 209 FP risk assessments, 66 (32\%) had other
Fig. 4 Respiratory rates in patient with a HF admission. From top to bottom: daily maximum, median and minimum respiratory rates. Thin lines with markers: daily trends; thick lines: 5-day moving averages Dotted line indicates the day of HF admission.

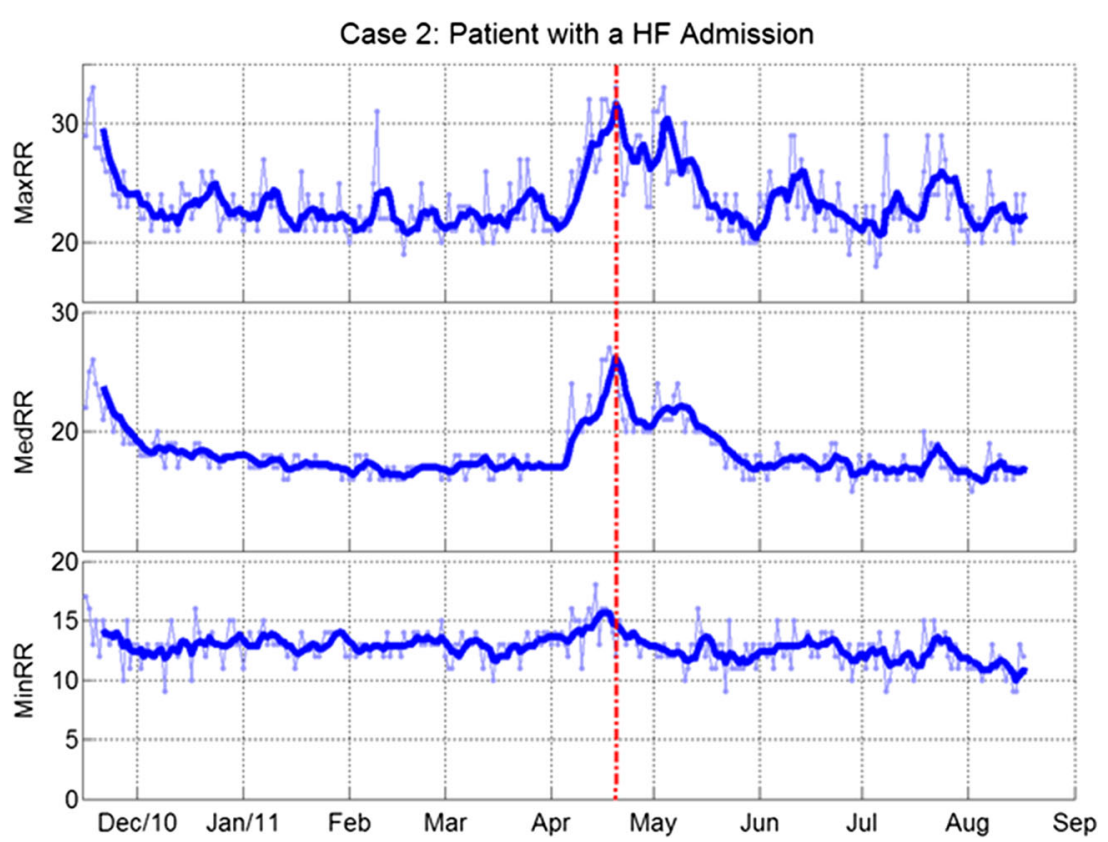


Fig. 5 Changes in respiratory rate metrics prior to a $\mathrm{HF}$ event
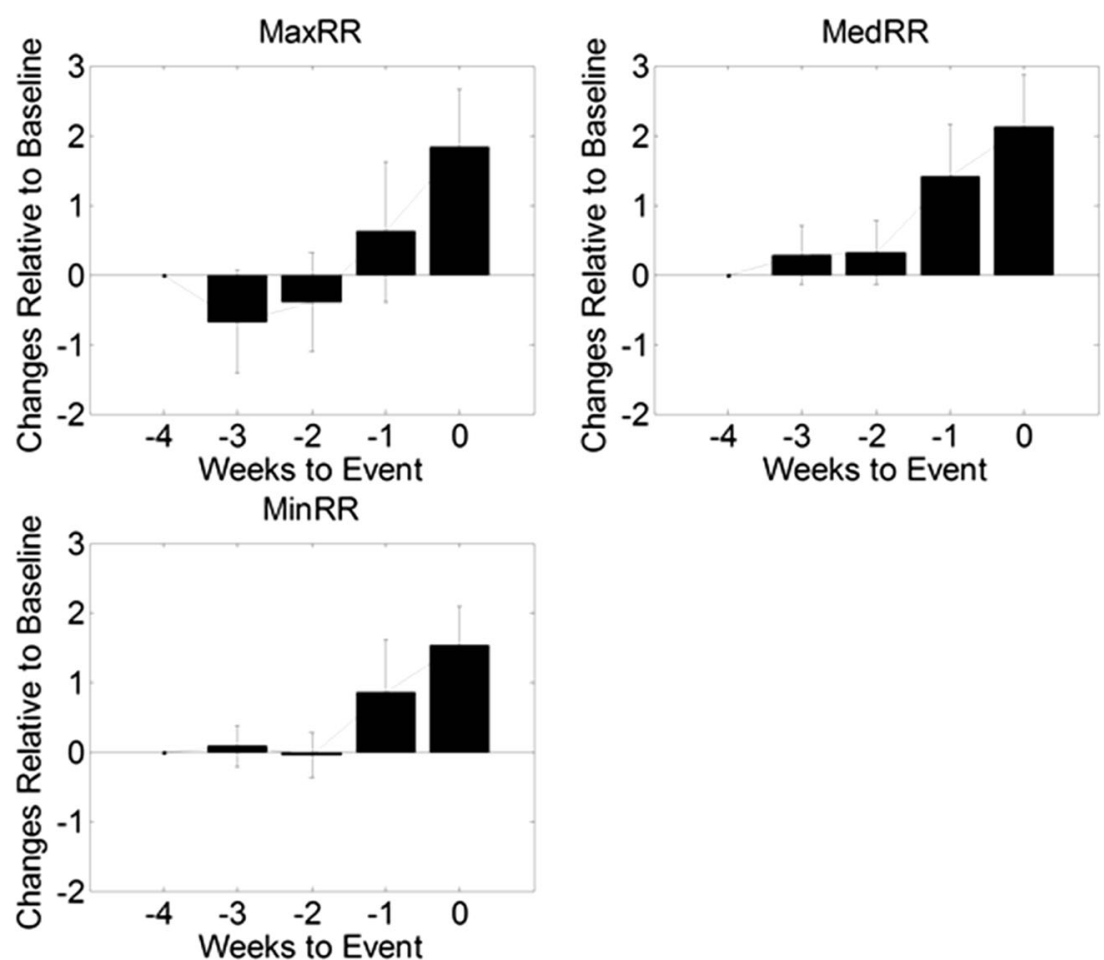

types of adverse events (AE) in either evaluation or observation window: $26(12 \%)$ had at least one non-HF AE in the observation window, $46(22 \%)$ had at least one $\mathrm{AE}$ in the evaluation window, and $6(3 \%)$ had AEs in both windows.

The stratification results suggested that patients with a high standard deviation in daily median RRT had a significantly increased risk of a HF event within the month following a risk assessment. However, the calculation of standard deviation may not be easy for clinicians when reviewing patient's longitudinal RRT data. We therefore evaluated an alternative way to estimate an effective data range by calculating the range of the data after removing the two largest and two smallest data points every month. With a threshold of $3 \mathrm{br} / \mathrm{min}$, the range also showed a strong stratification power to identify patients
Fig. 6 The results of the proportional means model of event-free time using standard deviation of respiratory rate during the evaluation periods

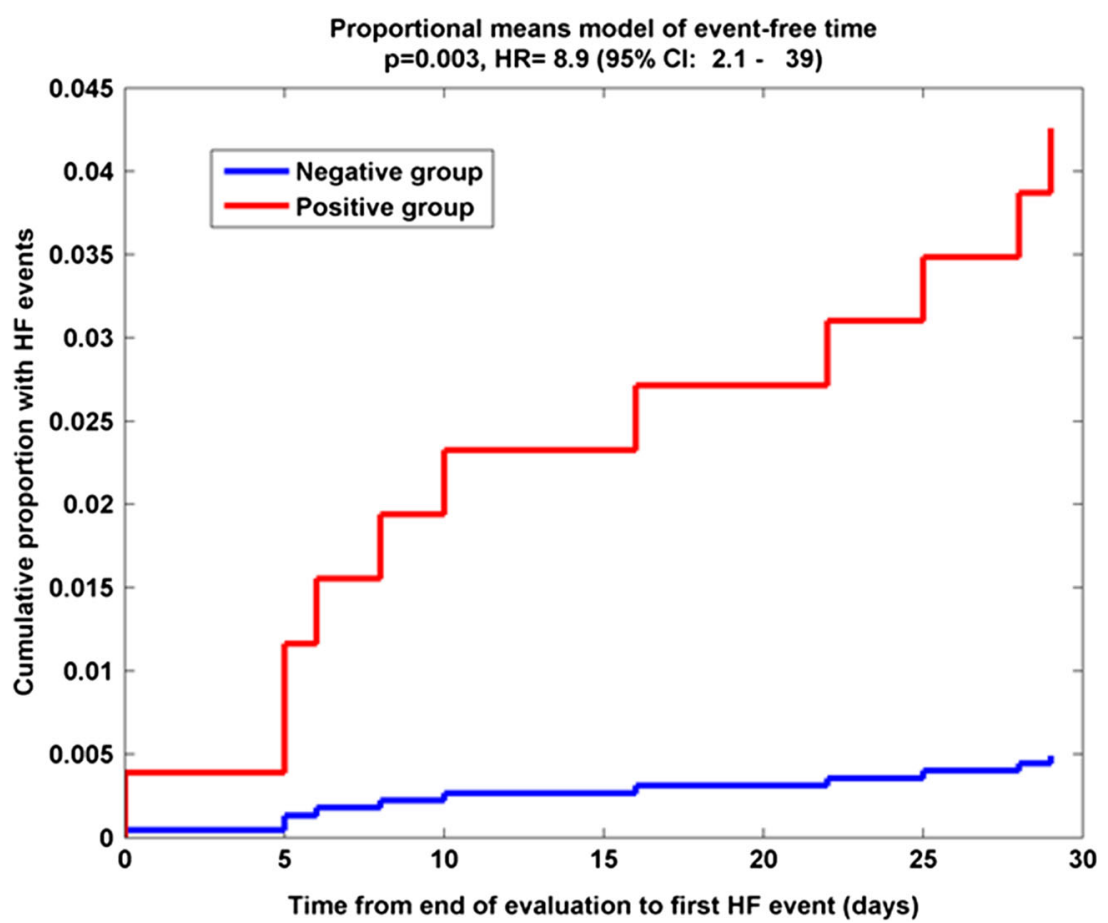


with high risk of HF events (HR=4.8, $95 \%$ CI 1.5-15, $p=$ 0.007). After adjusting for key clinical variables, the risk remained high $(\mathrm{HR}=7.3,95 \% \mathrm{CI} 2.3-24, p<0.001)$.

\section{Discussion}

We have evaluated changes in the ambulatory respiratory rate leading up to HF hospitalization. Daily maximum, median, and minimum respiratory rates were elevated during the 30day period leading up to HF events, indicating most patients experienced respiratory distress before HF admission. Page and colleagues reported elevated minute ventilation during rest and exercise observed prior to HF events [14]. However, changes in the respiratory rate were not reported. In our study, maximum respiratory rate showed a small drop 2-3 weeks before HF events (yet not statistically significant). This was mainly driven by a few patients whose maxRRs dropped due to a decrease in activity levels during this period. The activity level, however, was not statistically different prior to HF events. This observation was consistent with a larger study using similar device diagnostics in NYHA class I-IV patients with a CRT-D device [15]. Besides changes in respiratory rate, other metrics may also be used to quantify the trend leading up to HF events, such as slope of respiratory rate. We have previously reported that rates of change in daily maxRR, medRR, and minRR 30 days before HF events were significantly higher than that observed before a random time in the nonHF event group [16].

Of the six risk indices evaluated, only the standard deviation of daily median respiratory rates showed statistical significance in 30-day HF admission risk stratification. After adjusting for clinical variables that differed at baseline, patients with a higher daily variation in median respiratory rate still had a ninefold increased risk of experiencing a HF event within the next 30 days compared with those with a lower daily variation. This was consistent with the fact that standard deviation of medRR was also the only measure that was significantly higher in the group that experienced a HF event than the group that did not. Physiologically, medRR typically reflects the resting respiratory rate of the patient. A higher variation in medRR may indicate that the patient is not in a very stable condition, thus indicating a higher risk for worsening HF.

Based on a threshold of $1.0 \mathrm{br} / \mathrm{min}$ for the 30-day standard deviation of medRR, this metric correctly identified $73 \%$ of patient-observations with HF events (TPs), all of which had signs of pulmonary congestion or symptoms of shortness of breath. On the contrary, these signs and symptoms were absent in the three patient-observations followed by a HF event in the next 30-day that were not correctly identified (FNs). Clinically, these three patients did not suffer from shortness of breath, which is in line with the respiratory rate pattern recorded. However, in all three cases, individual weight measurements and device-based detection of arrhythmia burden might have been helpful to identify these patients, underscoring the importance of combining multiple measurements in heart failure patients.

Of all 800 patient observations, the numbers in the high and low risk groups were 27.3 and $72.7 \%$, respectively. The difference was more than threefold. Of those low-risk patientobservations, only three were followed by a HF event in the following month ( $99.7 \% \mathrm{NPV})$, indicating this metric has a very high negative predictive power. Since the trends are enabled by the implanted devices and available via remote monitoring system, they could be a useful screening tool to enable clinicians to identify patients who need closer monitoring, thus focusing limited healthcare resources on the patients who are at higher risk of decompensating. From a clinical perspective, remote monitoring of HF patients is transitioning from a "crisis prediction" regime to a "health maintenance" regime [17]. Using the negative predictive power of respiratory rate analysis in heart failure patients, this may be used in combination with other measures to distinguish stable patients from those at risk.

Approximately $95 \%$ of patient-observations in the high risk group did not experience a HF event during the following 30-day window. Of these, about $34 \%$ experienced other types of adverse events related to patient conditions either during the evaluation or the observation period. This indicated that respiratory rate is a very sensitive vital sign, but not specific to one pathological condition, just like any single sensor. Physicians need to incorporate respiratory rate with other clinical assessments before making a clinical decision or therapy adjustment.

Standard deviation is a statistical term that is easy to understand, but not necessarily easy to estimate when reviewing the trends. To translate it into a measure that is more easily assessed visually on a display, we evaluated the stratification ability of a range measure. A simple calculation of data range subtracting a minimal value from a maximal value is vulnerable to outliers, while the range after removing the two largest and two smallest values avoids the problem. Both range measures were able to identify patients at a higher risk of experiencing worsening HF in the next 30 days when medRRT had a range larger than $4 \mathrm{br} / \mathrm{min}$ in the 30-day evaluation window. The standard deviation had a better discriminatory power than the range measure. This is due to the resolution of the RRT metrics reported by the device (1 br/min), which limits the resolution of the range measure.

There have been a number of publications that evaluated the performance of combined diagnostics from implanted devices to stratify a short-term risk for HF hospitalization or readmission [18-22]. PARTNERS-HF [18] is a prospective study that evaluated the stratification power of combined device diagnostics, including fluid index, atrial fibrillation 
burden, bi-ventricular pacing, heart rate, and heart rate variability. Patients with a positive combined HF device diagnostics had a 5.5-fold increased risk of HF hospitalization with pulmonary signs or symptoms within the next month. A couple of recent publications confirmed the approach using Bayesian model [19] and showed value in 30-day readmission risk stratification using these diagnostics [20]. The best risk score from these studies yielded a HR of 10 for 30-day HF hospitalization when comparing high-risk group to low-risk group and a HR of 2.1 when comparing medium-risk to lowrisk. In our study, we were able to demonstrate a performance similar to the combined diagnostics using only respiratory rate trends, suggesting respiratory rate would add significant value in such an approach. Preliminary results from another study evaluating existing device diagnostics [21, 22] haven shown an improved risk stratification power combining respiratory rate with other device diagnostics such as pacing and shock lead impedance, activity, heart rate, atrial arrhythmia burden, weight, and \% biventricular pacing.

\section{Limitations}

The study is small, especially in terms of the number of HF events; the majority of the patients are NYHA class III; HF events were not adjudicated by an independent clinical event committee; and the analysis was retrospective in nature and was not tested in an independent data set. Larger studies are needed to validate these preliminary observations. While it is useful to have a good negative predictive marker, clinically, it is also relevant to have a consistent marker detecting early signs of worsening heart failure that could indicate a need for intervention to prevent hospitalization.

\section{Conclusions}

Daily maximum, median, and minimum respiratory rates are significantly elevated prior to HF events. Patients with a higher day-to-day variability in median respiratory rate were at increased risk of experiencing a HF event. Ambulatory daily respiratory rate trends may be a valuable addition to standard management for HF patients in order to pre-empt significant decompensation and hospitalization.

Acknowledgments The authors would like to thank all investigators in the NOTICE-HF study. The authors would also like to thank Elizabeth Scheurer for adjudicating all adverse events.

Conflict of Interests Yi Zhang, Qi An, Viktoria Averina, Ljubomir Manola Dries Kestens, and Nick Wold are full-time employees of Boston Scientific Corporation.

Funding The study was fully funded by Boston Scientific Corporation.

\section{References}

1. Braunwald, E., Colucci, W., \& Grossman, W. (1997). Clinical aspects of heart failure: high-output heart failure; pulmonary edema. In E. Braunwald (Ed.), Heart Diseases (5th ed., pp. 464-465). New York: WB Saunders.

2. Mancini, D. M. (1995). Pulmonary factors limiting exercise capacity in patients with heart failure. Progress in Cardiovascular Diseases, 37, 347-370.

3. Fonarow, G. C., \& ADHERE. Scientific Advisory Committee. (2003). The Acute Decompensated Heart Failure National Registry (ADHERE): opportunities to improve care of patients hospitalized with acute decompensated heart failure. Reviews in Cardiovascular Medicine, 4(Suppl 7), S21-S30.

4. Ekman, I., Cleland, J., Swedberg, K., Charlesworth, A., Metra, M., \& Polle-Wilson, P. (2005). Symptoms in patients with heart failure are prognostic predictors: insights from COMET. Journal of Cardiac Failure, 11, 288-292.

5. Silva, L., Mielniczuk, L., Laberge, M., Anselm, A., Fraser, M., Williams, K., \& Haddad, H. (2004). Persistent orthopnea and the prognosis of patients in the heart failure clinic. Congestive Heart Failure, 10, 177-180.

6. Rame, J. E., Sheffield, M. A., Dries, D. L., Gardner, E. B., Toto, K. H., Yancy, C. W., \& Drazner, M. H. (2001). Outcomes after emergency department discharge with a primary diagnosis of heart failure. American Heart Journal, 142, 714-719.

7. Lee, R. S., Austin, P. C., Rouleau, J. L., Liu, P. P., Naimark, D., \& Tu, J. V. (2003). Predicting mortality among patients hospitalized for heart failure. Journal of the American Medical Association, 290, 2581-2587.

8. Charach, G., Rabinovich, P., Grosskopf, I., \& Weintraub, M. (2001). Transthoracic monitoring of the impedance of the right lung in patients with cardiogenic pulmonary edema. Critical Care Medicine, 29, 1137-1144.

9. Yokoyama, H., Sato, H., Hori, M., Takeda, H., \& Kamada, T. (1994). A characteristic change in ventilation mode during exertional dyspnea in patients with chronic heart failure. Chest, 106, 1007-1013.

10. Weber, K. T., Kinasewitz, G. T., Janicki, J. S., \& Fishman, A. P. (1982). Oxygen utilization and ventilation during exercise in patients with chronic cardiac failure. Circulation, 65, 1213-1223.

11. Clark, A. L., Volterrani, M., Swan, J. W., \& Coats, A. J. (1997). The increased ventilatory response to exercise in chronic heart failure: relation to pulmonary pathology. Heart, 77, 138-146.

12. Duguet, A., Tantucci, C., Lozinguea, O., Isnard, R., Thomas, D., Zelter, M., Derenne, J. P., Milic-Emili, J., \& Similowski, T. (2000). Expiratory flow limitation as a determinant of orthopnea in acute left heart failure. Journal of the American College of Cardiology, 35, 690-700.

13. Lin, D. Y., Wei, L. J., Yang, I., \& Ying, Z. (2000). Semiparametric regression for the mean and rate function of recurrent events. Journal of the Royal Statistical Society, Series B: Statistical Methodology, 62, 711-730.

14. Page, E., Cazeau, S., Ritter, P., Galley, D., \& Casset, C. (2007). Physiological approach to monitor patients in congestive heart failure: application of a new implantable device-based system to monitor daily life activity and ventilation. Europace, 9, 687-693.

15. Gilliam, F. R., Ewald, G. A., \& Sweeney, R. J. (2012). Feasibility of automated heart failure decompensation detection using remote patient monitoring: results from the decompensation detection study. Journal of Innovations in Cardiac Rhythm Management, 3, 1-10.

16. Goetze, S., Lambiase, P., Schilling, R., Trappe, H., Winter, S., Zhang, S., Wold, N., Manola, L., Kestens, D. (2012). Rate of change in respiratory rate is significantly higher in patients with $\mathrm{HF}$ events than patients without HF events, International symposium on progress in clinical pacing.

17. Cleland, L. G. F., \& Antony, R. (2011). It makes SENSE to take a safer road. European Heart Journal, 32, 2225-2227. 
18. Whellan, D. J., Ousdigian, K. T., AI-Khatib, S. M., Sarkar, S., Porter, C. B., Pavri, B. B., O'Connor, C. M., \& PARTNERS Study Investigators. (2010). Combined heart failure device diagnostics identify patients at higher risk of subsequent heart failure hospitalizations. Journal of the American College of Cardiology, 55, 18031810.

19. Cowie, MR., Sarkar, S., Koehler, J., Whellan, DJ., Crossley, GH., Tang, WH., Abraham, WT., Sharma, V., Santini, M. (2013). Development and validation of an integrated diagnostic algorithm derived from parameters monitored in implantable devices for identifying patients at risk for heart failure hospitalization in an ambulatory setting. European Heart Journal, 34(31), 2472-2480.
20. Whellan, D. J., Sarkar, S., Koehler, J., Small, R. S., Boyle, A., Warman, E. N., \& Abraham, W. T. (2013). Development of a method to risk stratify patients with heart failure for 30-day readmission using implantable device diagnostics. American Journal of Cardiology, $111,79-84$.

21. Cao, M., Gold, M. R., Zhang, Y., Wold, N., Wehrenberg, S., An, Q., \& Boehmer, J. (2014). Implantable device diagnostics identify patients at higher risk of heart failure events in 30 days, Heart \& Lung. The Journal of Acute and Critical Care, 43(4), 381-382.

22. Molon, G., Capucci, A., Zhang, Y., Wold, N., An, Q., Wehrenberg, S., \& Boehmer, J. (2014). Existing device diagnostics identify patients at higher risk of worsening heart failure in 30 days. Europace, 16(Suppl 2), ii123. 